co-operation. Books and journals, including technical publications for scientific men, others that will interest the " average man" in science and in the wider application of the scientific method, are leading agencies. Science Service, for which the American Association shares the responsibility, should be made a large factor in the dissemination of science. But of all agencies for the promotion and diffusion of science, scientific societies are the most important-the national societies in each science, the state and local academies, most of all the association in which these are united and which at its annual meetings.and at all times advances the interests of science and of scientific men and impresses on the widest public the weight and magnitude of modern science.

In spite of the limited value for science of direct introspection, our mental life is part of the real world, and is that part which is of the greatest concern to each of us. It may be, as has been suggested, that psychology lost its soul long ago and is now losing its mind ; but it cannot lose consciousness. Our perceptions, thoughts, intentions, and feelings are not only elements in sensori-motor arcs; for us they are also the end to which the whole creation moves. So far as production goes, consciousness may be only a spectator; but it is the ultimate consumer. We shall have in due time a scientific psychology of human welfare, of the things that are beautiful, good, and true. But it will not come until we get these things instead of talking about them; for science has meaning and value only in its usefulness. Psychology may supply economic values equal to those of the physical and biological sciences, human values of even greater significance.

Scientific research and the applications of science in the course of 150 years have increased fourfold the productivity of labour; they have doubled the length of life. Science has made it possible for each to work at routine tasks half as long as formerly and at the same time to consume twice as much wealth as formerly. Fourteen hours of labour, shared by women and children, once provided hovels, lice, and black bread for most people, luxury for a few. Seven hours of labour will now supply comfortable homes, warm clothes, and healthful food for all. If the resources provided by science were properly distributed-as they will be when we have an applied science of psychology-there is now sufficient wealth to enable all to share in the desirable luxuries that science has created, and to enjoy in full measure the most nearly ultimate goods of life-home, friends, things to do, freedom, self-respect.

The better lives secured through the increased wealth provided by science, together with the applications of science to hygiene, medicine, and surgery, have doubled the length of life. In the nations of the west, pestilence and famine have lost most of their terrors; of the three evil fates, only war survives from a pre-scientific and barbarous past. Much is crude and ugly in the modern world ; atrophied instincts and aborted impulses must be replaced by the products of a science of psychology before living can become free and fine. But those who call our industrial civilisation materialistic and ignoble have narrow thoughts and scant idealism. They fail to imagine what it means in terms of love and suffering that of ten infants born, formerly only two or three survived childhood, while now eight or nine may live to have children of their own.

The applications of science have abolished slavery and serfdom, the need of child labour, the subjection of woman; they have made possible universal education, democracy, and equality of opportunity, and have given us so much of these as we have. Science has not only created our civilisation; it has given to it the finest art and the truest faith. The advancement of science should be the chief concern of a nation that would conserve and increase the welfare of its people.

\title{
The Progress of Directional Wireless Communication.
}

\section{By Dr. R. L. Smith-Rose.}

$\mathrm{F}$ ROM the earliest days of practical wireless communication, it was realised that one of the most important services which this art would render to humanity was its contribution towards the safety of life at sea. The possibility of a ship being able to keep in communication with the remainder of the world during the longest voyages, and, in time of distress, to broadcast an appeal for assistance, has removed much of the terror of shipwreck and fire at sea. A later development, which bids fair to rank second in importance to the above, is the direct application of wireless to aerial and marine"navigation. There are, broadly, two methods by which a wireless signal may be used to determine the direction of any given fixed point. In the first, the direction of arrival of the signals from any transmitting station is determined by means of a direction-finder. The second method involves the transmission of a rotating beam of large or small angle and its reception on any suitable receiver.

The wireless direction-finder makes use of the fact that in electromagnetic waves travelling over the earth's surface, the magnetic force of the waves is horizontal and perpendicular to the direction of transmission. If a closed loop or coil of one or more turns with its plane vertical is used to receive such waves, the strength of the signal E.M.F. induced in the loop will be proportional to the cosine of the angle between the plane of the loop and the direction of arrival of the waves. Hence, if the loop is rotated about a vertical axis, the strength of the signals received will pass through successive maximum and zero values at intervals of $90^{\circ}$. A consideration of the case shows that the rate of change of the resulting signal strength is greatest when passing through the zero position, which is therefore the most sensitive condition for the determination of the direction of arrival of the waves. In this zero position the plane of the loop is perpendicular to the direction of the incoming waves and thus of the transmitting station.

There are three practical forms of wireless directionfinder in use to-day operating on this fundamental principle of the simple rotating loop: and it can be shown, both theoretically and experimentally, that the accuracy is of the same order for all the systems, each of which has its own field of application. The

NO. 2933, VOL. II 7 ] 
limiting accuracy to which the modern direction-finder has been developed is about $\mathrm{r}^{\circ}$, which, it may be remarked, is ample for the present-day needs of navigation.

Apart from any defects of instrument design and construction, the direction-finder is subject to errors which are conveniently divided into three classes. In the first class are those due to local conditions surrounding the instrument, such as metal-work, overhead wires, and trees. The currents set up in such objects create a secondary field in the neighbourhood which is superimposed on the primary field of the waves, producing a resultant field the direction of which may deviate seriously from that of the original field. It is usual in selecting a site for a wireless direction-finder on land to attempt to avoid such sources of error, but it is seldom that an ideal site can be found. The small residual errors which are experienced are taken into account by calibrating the station on various fixed transmitting stations. Instances in which an error due to the site of the installation is unavoidable are those of ships and aircraft. In these cases the error in apparent direction varies in an approximately sine-wave form with the direction of the incoming waves relative to the fore-andaft line of the ship. In such installations, either a correction curve is prepared from observations taken while the ship is swung on a fixed transmitting station, or the error is approximately compensated for by a suitable circuit arrangement.

The second class of error is in the nature of a surface refraction effect and is encountered when the path of the waves lies approximately parallel to the boundary between land and sea. If observations are made on wireless waves crossing a coast-line, the direction-finding instrument indicates that the waves in crossing from sea to land experience a deviation in a direction towards the normal to the coast-line at the point of transit. No satisfactory theoretical explanation of this phenomenon has yet been given, but as the error is frequently of the order of $5^{\circ}$, it is customary to avoid, wherever possible, the taking of bearings on waves travelling in a direction approaching that of a coast-line.

The type of error in the third class presents more difficulty in accurate work, on account of its very erratic and variable behaviour. Fortunately this class of variable error is encountered only when the range of transmission exceeds certain minimum values, beyond which the apparent direction of arrival of the waves is subject to somewhat erratic variations, the frequency and magnitude of which are dependent to a limited extent upon the wave-length employed, the distance of transmission, and also upon the season and time of the day at which the observations are made. During the hours of full sunlight in summer, these variable errors have a maximum value of about $4^{\circ}$. As sunset is approached they increase in frequency and magnitude, and from about one hour before sunset until about one hour after sunrise the apparent direction of arrival of the waves varies rapidly on either side of the true direction over an arc which is often $10^{\circ}$ or $20^{\circ}$ and may occasionally exceed $90^{\circ}$.

The theory which has been developed to explain these errors rests on the same basis as the theory of the fading of wireless signals and of the mode of propagation of wireless waves around the earth's surface. This theory assumes that the upper ionised regions of the atmosphere are operative in deflecting wireless waves arriving from a transmitting station in such a manner that they are eventually returned to the earth's surface. A direction-finding station will thus receive waves from the transmitter not only horizontally along the earth, but also from a downward direction. If, as is possible from several causes, the plane of polarisation of the downcoming waves differs from that normally encountered in transmission along the earth's surface, the direction of the horizontal component of the resultant magnetic field due to the two arriving waves will in general not be at right angles to the direction of transmission. Thus, although there has been no departure of the line of travel of the waves from the vertical plane connecting transmitter and direction-finder, the latter will indicate an error in the apparent direction of arrival. This theory is being supported by the accumulation of a large and varied amount of experimental evidence, and the investigations concerned provide an excellent means of studying the physics of the upper atmosphere.

\section{DiRECTION-FINDERS IN NAVIGATION.}

The discovery of the existence of these errors in the course of the development of direction-finding during the War inspired a lack of confidence in this art among those responsible for marine navigation. Systematic research carried out during the last few years has, however, brought to light a state of affairs which has resulted in the reinstatement of the wireless directionfinder as a direct aid to seamen. While the instrument may be used at ranges of several hundred miles to give a general idea of a ship's position, it is in the making of a landfall after an open-sea voyage, or in the navigation of harbour entrances during bad or foggy weather, that the wireless direction-finder is in demand. The ranges at which it is required to operate with accuracy are seldom greater than 50 miles, and never greater than roo miles. Now the variable errors described above are only experienced when the range of transmission exceeds 30 miles overland, or 80 to roo miles oversea. Hence, by a suitable choice of shore transmitting stations, the ship's direction-finder can be used to operate on waves travelling entirely over sea, with the result that, within its required range, about 95 per cent. of the observed bearings fall within a $2^{\circ}$ limit of error, which is found to be adequate for most navigational purposes.

During several years' observations, no effect of fog or other surface weather conditions has ever been noticed in wireless direction-finding, which is again fortunate for its use in navigation during times when visual observations are impracticable.

In the application of the direction-finder to aircraft, similar remarks apply as to its accuracy, with one important addition. When the direction-finder is operated on the ground on the transmissions from a machine in the air, the observed bearings are liable to error when the angle of elevation of the machine at the ground station exceeds a small value. This error is caused by the operation of downcoming waves on the direction-finder, but is evidently only appreciable for very short range transmission. The limited amount of experience with direction-finders used in

$$
\text { NO. 2933, VOL. I I 7] }
$$


the aircraft itself appears to show that these are destined to be of great service in the future of aerial navigation.

Turning now to other means by which directional wireless may be used in connexion with navigation, we have first the scheme which is practically an inversion of the above systems; that is, a rotating loop or its equivalent is used at the transmitting end and the reception of the signals is carried out on an ordinary aerial. When a closed loop or frame coil with its plane vertical is used for transmission purposes, the variation along the earth's surface is a maximum along a line through the plane of the coil and decreases according to a cosine law to a zero value along the axis of the coil. If, therefore, the coil is rotated about a vertical axis, the strength of signals received at a distance on an ordinary aerial will vary from a maximum when the coil is pointing towards the receiver to a minimum or zero value when it is at right angles thereto. In the practical operation of the device the coil transmitter is rotated at some convenient uniform rate, and arrangements are made for characteristic signals to be transmitted as the coil passes through two positions such as the north-south and east-west lines. By the use of a stop watch at the receiver, the times of rotation of the coil from one of these positions to the minimum signal position can be determined, together with the time of complete rotation. A simple calculation then serves to obtain the direction of the transmitting beacon from the receiver.

The great advantage of this system is that any ship or aircraft which is provided with a wireless receiver can obtain directional observations on these transmitters, and if a suitable distribution of the latter could be arranged, the method would be very useful for navigational purposes. Although it was developed in Germany before the War and an organisation of rotating beacon transmitters was set up, the system has of recent years only been used for experimental purposes, and its commercial application has still to be realised. It may be mentioned here that both this type of directional transmitter and the directional receiver referred to previously can be provided with a simple circuit modification which results in only one signal minimum being produced in a rotation of the coil through $360^{\circ}$. This "sense-finding" attachment results in the removal of the $180^{\circ}$ ambiguity which is otherwise inherent in these methods.

The development and use of the above directional systems has been carried out on the wave-lengths chiefly used for communication purposes in aerial and marine. wireless, i.e. approximately from 300 to 3000 metres. As a result of the development of the thermionic valve to a high degree for both transmission and reception purposes, it has become possible to extend the practice of wireless communication to very much shorter wave-lengths. Among the numerous vistas of development thereby opened up, not the least important is the practicability of resorting to the use of reflectors for concentrating the radiated energy in the required direction of transmission. By the use of suitable reflectors at both the transmitting and receiving end of a point-to-point communication link, the resulting concentration of wireless wave energy can be made equivalent to a magnification of several thousand times as compared with the ordinary transmitter which radiates its energy equally in all directions. This advantage, combined with the inherent properties which waves lying within certain bands of wave-lengths between $\mathrm{I}_{5}$ and 100 metres appear to possess for travelling through the upper atmosphere to great distances with very small attenuation, has already caused a revolution in ideas as to longdistance wireless communication. The resulting opening-up of a large number of fresh communication channels has come as a great boon to wireless engineers struggling with an already congested ether.

\section{The Wireless Beacon.}

By the use of waves of length still shorter than those mentioned, it becomes possible to utilise another principle in applying directional wireless to navigation purposes. This consists in combining the aerial system of a wireless transmitter with a suitable reflector so that a fairly concentrated beam of wireless waves is sent out in one direction, which is varied by the regular rotation of the whole arrangement. We have here literally a wireless lighthouse, since it is exactly analogous to the rotating beam of light emitted by certain lighthouses around our coasts. Now it is a well-known optical law that for a reflector to be effective its dimensions must be at least comparable with the wave-length. Hence it is evident that for the rotation of the reflector to be at all practicable, the wave-length employed must be quite small.

In the two revolving beam transmitters which have been set up in the last year or two, the wave-length employed is about 6 metres. In the first station erected at Inchkeith, Firth of Forth, the aerial is a straight vertical wire erected in the focal line of a cylindrical parabolic reflector system of vertical wires. In the more recent station erected at the South Foreland, the aerial system comprises a number of energised aerials arranged in a plane, and in front of a similar parallel system of reflecting wires. The result in each case is the transmission of a beam of limited angle which is rotated uniformly at a rate of about one revolution per minute. Any ship fitted with a suitable receiver for the wavelength employed will then only receive signals as the beam flashes past its aerial. From the code signals heard the direction of the beam at that moment may be read from a simple chart. The range of the station is necessarily somewhat limited, owing to the great absorption of energy of such short waves by the earth. In experiments carried out a short time ago, it was claimed that a maximum range of 100 miles oversea could be obtained.

A great advantage possessed by this system over those previously mentioned is that it should be free from any errors due to its waves being deflected from the upper atmosphere. This fact may prove to be very important in securing a practical working range well in excess of that given by the waves travelling along the earth's surface. An advantage of the use of the short wave-length is the comparative freedom from interference which is experienced at the recerver. On the other hand, the use of such short waves may result in serious errors due to the coastal refraction effect previously mentioned, and to deviation of the waves by such obstacles as cliffs and buildings acting as reflectors. 